\title{
Comparing Fluorescence and ToF-SIMS Imaging of DNA Microarray Spots
}

\author{
Líney Árnadóttir,* Nicolas Vandencasteele, ${ }^{* *}$ David W. Grainger,*** and Lara Gamble, ${ }^{* *}$ \\ * Department of Chemical Engineering, University of Washington, Seattle, WA 98195 \\ ** Department of Bioengineering, University of Washington, Seattle, WA 98195 \\ *** Department of Pharmaceutics and Pharmaceutical Chemistry, University of Utah, Salt Lake \\ City, UT 84112
}

DNA microarrays have commercial potential both as point-of-care rapid diagnostic tests and in genetic screening, as well as basic research applications. However, lack of assay signal reliability and reproducibility has contributed to lack of regulatory approval for clinical use of many microarray chemistries. DNA microarrays are often made by microprinting, where a nanoliter of DNA solution is printed onto functionalized glass surfaces where the droplet rapidly evaporates, producing a dried spot. The printed slides are exposed to biological samples and then most commonly analyzed by fluorescence imaging. Both intra-spot and spot-spot optical heterogeneity is common but fluorescence mapping provides no chemical or structural information for these effects.

We have used time-of-flight secondary ion mass spectrometry (ToF SIMS) imaging and both x-ray photoelectron (XPS) spectroscopy and XPS imaging to better understand the heterogeneity of these DNA microarray spots. The DNA microarrays are printed following a previously published procedure [1] and fluorescence imaging data are acquired. All XPS analyses were done in a Kratos Axis Ultra DLD XPS. ToF-SIMS data were acquired on an IONTOF ToF SIMS 5-100 instrument using $\mathrm{a} \mathrm{Bi}_{3}{ }^{++}$primary ion source. Principal component analysis (PCA) was applied to the data as part of the ToF-SIMS data analyses as described previously [2]. Single-stranded amine-terminated 40mer DNA probes are spotted onto amine-reactive microarray slides. Three different DNA probe concentrations were printed $(10,20$ and $40 \mu \mathrm{M})$ and, within each print concentration, the ratio between Cy3-labeled DNA and identical unlabeled DNA was varied from 0, 25, 75, and 100\%.

Fluorescence data clearly show non-uniformity of dried microarray spots. The ToF-SIMS imaging for fragments originating in the DNA backbone such as $\mathrm{PO}_{2}{ }^{-}, \mathrm{PO}_{3}{ }^{-}$and $\mathrm{Na}_{2} \mathrm{PO}_{3}{ }^{+}$shows a similar pattern. Fragments originating from the $\mathrm{Cy} 3$ dye label show the same trend as well, suggesting even distribution of Cy3 dye label within the DNA, but a non-homogeneous distribution of printed DNA in the spot after drying.

Figure 1 shows corresponding fluorescence and ToF-SIMS images of spots with three different DNA probe concentrations, all with 75\% Cy3 dye labeling. ToF-SIMS images show that the heterogeneity in the fluorescence images is not due to uneven distribution of the Cy3 labeled DNA within the spot but due intrinsically to the DNA. Figures 1 and 2 show how the spot non-uniformity changes significantly with DNA probe concentration but varies little with Cy3 label concentration, suggesting that the dye label does not significantly affect spot uniformity.

ToF-SIMS images can provide valuable chemical information that complements the fluorescence image analysis of printed microarray chemistry. Comparison of corresponding ToF-SIMS images and fluorescence data shows that spot non-uniformities are due to the DNA and not the Cy3 labeling. DNA concentration in the probe print solutions has a significant effect on the spot uniformity: spots 
become less uniform at higher print concentrations. This non-uniformity results from rapid spot drying not allowing equilibrium mixing or uniform interactions with the surface.

[1] C.Y. Lee et al., J. Am. Chem. Soc. 129 (2007) 9429.

[2] M.S. Wagner et al., Appl Surf. Sci. 252 (2006) 6575.

[3] This research was supported by NIH grants EB-002027, and EB-006163

$10 \mu \mathrm{M}, 75 \%$ Cy3
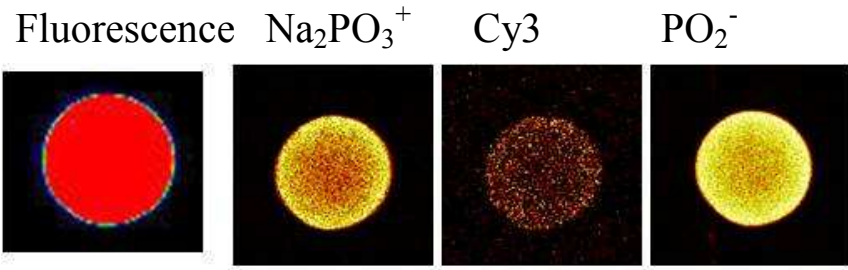

$\mathrm{PO}_{3}^{-}$

$20 \mu \mathrm{M} 75 \% \mathrm{Cy} 3$
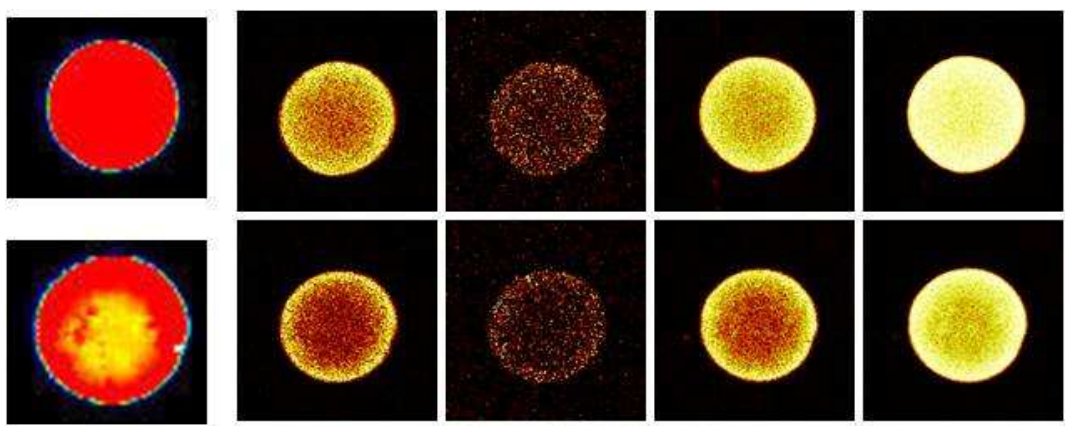

$40 \mu \mathrm{M} 75 \% \mathrm{Cy} 3$
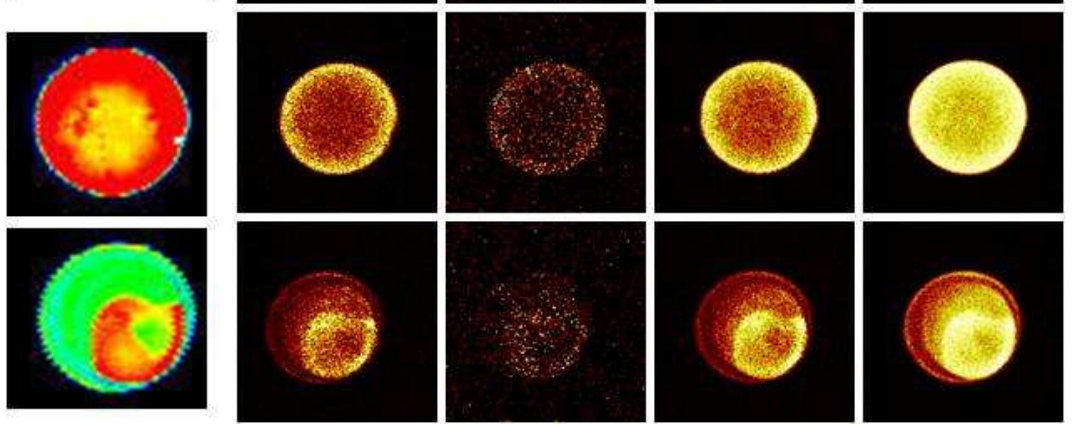

FIG. 1. DNA spot images compared for fluorescence and ToF-SIMS imaging. Three rows show the same percentage of Cy3 DNA label (75\%) but at three different DNA probe concentration $(10,20$, and $40 \mu \mathrm{M}$ ). Fluorescence images are in the first column followed by their ToF-SIMS images, two from the positive spectra $\left(\mathrm{Na}_{2} \mathrm{PO}_{3}{ }^{+}\right.$of the DNA backbone and $\mathrm{C}_{12} \mathrm{H}_{10} \mathrm{~N}$ from the $\mathrm{Cy} 3$ dye), and two from the negative spectra $\left(\mathrm{PO}_{2}{ }^{-}\right.$and $\mathrm{PO}_{3}{ }^{-}$both originating from the DNA backbone). All rows show a similar patterns, suggesting even distribution of the Cy3 label within the DNA microarray spot.

$40 \mu \mathrm{M}$

Fluorescence

$\mathrm{Na}_{2} \mathrm{PO}_{3}{ }^{+}$

$\mathrm{PO}_{2}^{-}$
$0 \%$
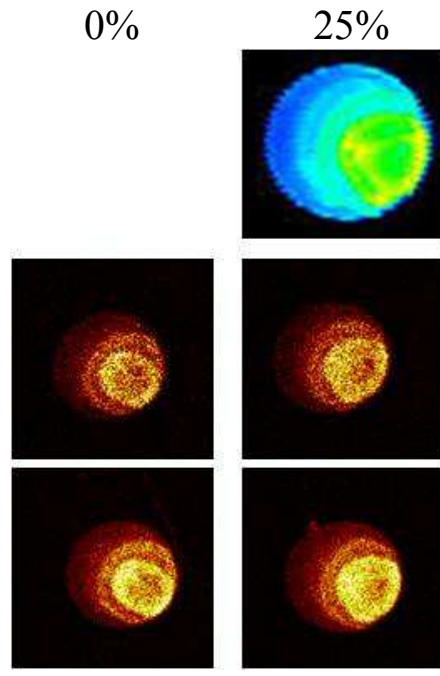

$75 \%$
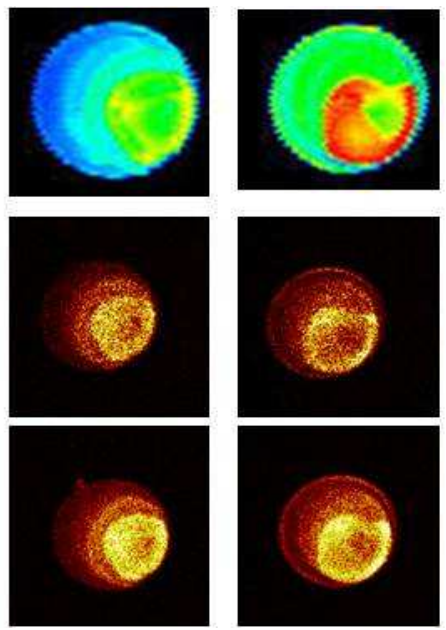

$100 \%$
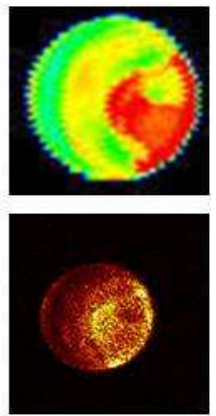

FIG. 2. Fluorescence (top row) and ToF-SIMS images of identical printed DNA spots for $40 \mu \mathrm{M}$ DNA probe concentrations at different $\mathrm{Cy} 3$ label concentrations. 